\title{
Simplified Sensorless Torque Estimation Method for Harmonic Drive Based Electro-Mechanical Actuator
}

\author{
Mohamed A. A. Ismail ${ }^{\circledR}$, Jens Windelberg ${ }^{\circledR}$, and Guangjun Liu ${ }^{\circledR}$, Senior Member, IEEE
}

\begin{abstract}
Torque measurements can significantly enhance control and monitoring loops for many mechatronic and aerospace applications. A typical challenge is to justify incorporating a torque sensor in terms of cost, system complexity, and reliability. Recently, sensorless torque estimation methods have been developed for robotic joints that include harmonic drive transmissions (HDTs). The principle is based on their relatively low torsional stiffness, which allows for estimating the transmitted torque by measuring the torsional angles (via existing joint encoders) and a compliance model. However, these methods are based on nonlinear models that are difficult to identify and tune. In this study, a simplified torque estimation method is introduced based on the structural damping friction of the HDTs. The structural damping can be correlated to the HDT torque using a simplified linear dynamic model and torsional rate measurements. Experimental results have validated the proposed method, using a robotic joint setup with an external torque sensor that has been previously utilized for testing several torque estimation methods.
\end{abstract}

Index Terms-Robot, joint torque estimation, harmonic drive, electro-mechanical actuator.

\section{INTRODUCTION}

A HARMONIC drive transmission (HDT), also known as strain wave gearing, is a compact gear mechanism widely used for actuating robotic joints and in aerospace applications [1]-[3]. HDT-based electro-mechanical actuators, comprising an electrical motor and HDT, provide a superior power-to-weight ratio, coaxial design, and backlash-free transmission [1], [2]. Another feature of HDTs is they allow for the possibility of estimating the transmitted torque without using a force sensor. Conventional force sensors, in addition to having narrow bandwidth, add an undesired structural load [4]. Inherent torque sensing is crucial for many motion control applications in robotics [2]-[4], such as joint torque feedback [2] and collision detection [3]. Recently, torque sensing of HDTs has been integrated for smart flight control actuators to measure the aerodynamic load and significantly enhance fault detection for safety-critical systems [6].

Manuscript received October 12, 2020; accepted January 2, 2021. Date of publication January 18, 2021; date of current version February 2, 2021. This letter was recommended for publication by Associate Editor G. Huang and Editor L. Pallottino upon evaluation of the reviewers' comments. This work was supported by the TEMA-UAV project through the German National Aerospace Research Program (Lufo V-3), and in part by the Natural Sciences and Engineering Research Council of Canada (NSERC). (Corresponding author: Mohamed A. A. Ismail.)

Mohamed A. A. Ismail and Jens Windelberg are with the DLR German Aerospace Center, Institute of Flight Systems, 38108 Braunschweig, Germany (e-mail: mohamed.ismail@dlr.de; jens.windelberg@dlr.de).

Guangjun Liu is with the Department of Aerospace Engineering, Ryerson University, Toronto, ON M5B 2K3, Canada (e-mail: gjliu@ryerson.ca).

Digital Object Identifier 10.1109/LRA.2021.3052392
The torque sensing principle is based on the low torsional stiffness of HDTs due to an internal elastic element called flexspline. A torsional deformation of the Flexspline can be measured and mapped to the corresponding HDT torque by two main approaches [2]-[8]. The first approach utilizes strain gauge sensors mounted on the flexspline to measure the torsion deformation and the transmitted torque through a direct calibration process [7], [8]. This approach achieves good estimation results, but it requires an additional strain gauge sensor and signal conditioning hardware. The second approach is based on measuring flexspline torsion with two high-precision angular encoders at the input and output shafts of an HDT [2]-[5]. The encoder measurements are fed to a compliance model to estimate the applied torque. This approach is a typical sensorless torque estimation concept because these encoders are already integrated into many robotic and motion control architectures. Recent studies for sensorless torque estimation include Vassileva et al. [3], Zhang et al. [2], and Xia et al. [5].

Vassileva et al. [3] investigated a torque estimation method based on the torque disturbance observer studied in [4]. The observer does not exploit HDT compliance; nevertheless, it uses the frictional torque of the HDT in terms of Coulomb and viscous friction. The estimated torque is obtained from the velocity response of the HDT. The results have been experimentally validated using HDT actuators in a five degree-of-freedom chopsticks robot. However, the torque estimation is limited at low velocities and with velocity reversal because a discontinuous model transition exists for separate friction models for positive and negative directions.

Zhang et al. [2] developed a torque estimation method based on HDT compliance for a robot joint. High-precision encoders at the input/output shafts of an HDT joint are used to measure the torsional deformation. The torsional deformation has been processed to separate torsional sub-deformations that are not correlated with the output torque of the HDT. Examples of these sub-deformations are kinematic error and hysteresis. Zhang et al. used torque measurements from the motor (i.e., input torque to HDT) to estimate and isolate a torsion error due to the HDT's hysteresis.

Xia et al. [5] reported significant torque errors for the torque estimation method previously developed by Zhang et al. in [2]. Xia et al. justified this deficiency because the compliance model in [2] cannot fully characterize the underlying torsion-torque dynamics. Xia et al. developed an enhanced torque estimation method based on the earlier model in [2], in addition to a compensation term based on Gaussian process regression (GPR). 


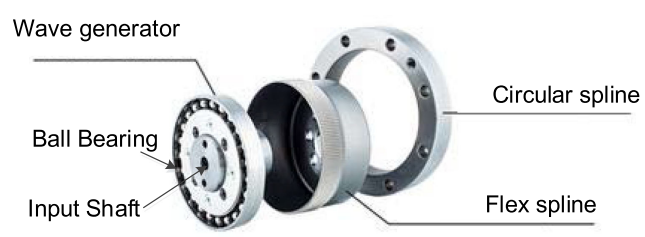

Fig. 1. Basic harmonic drive transmission assembly.

The GPR is a supervised learning technique that was used to model the unmapped dynamics in the model in [2] through a data training process. Enhanced torque sensing results have been achieved using torsional data measured by two high precision encoders, motor current measurements, and a full calibration test to tune the GPR structure.

In practical terms, the method reported by Xia et al. [5] is complicated for many applications because it includes physical as well as data-based torque estimation methods. Many applications have limited computational resources that are mainly for primary motion control functions. In addition, the elasticity of an HDT is typically subjected to normal aging, and the torque estimation methods will need frequent calibrations over the service life of the HDT.

In this study, a simplified torque estimation method is introduced based on the structural damping of HDT. The structural damping is identified by the torsional rate rather than the static torsion form used in the prior studies. The HDT torsion is calculated in a manner similar to the existing methods, by the angular encoders at the input/out shafts of the HDT, and a numerical differentiator is used to convert the torsion data into the torsional rate. A simplified linear dynamic model is empirically identified for estimating the HDT torque based on the torsional rate measurements. This new method will provide advantages for realizing reliable and computationally simple torque sensing capabilities for many existing and future robotic and aerospace applications.

The rest of the paper is organized as follows. Section II discusses a physical HDT model for torque estimation. The principle and calculation steps for the proposed torque estimation method are presented in Section III. Experimental setup and results are described in Section IV. Research contributions and future work are concluded in Section V.

\section{HARMONIC DRIVE TRANSMISSION}

The basic assembly for an HDT consists of three main components: the wave generator, the flexspline, and the circular spline, as shown in Fig. 1. There are different mounting configurations, and here, we consider the most common case in which the circular spline is fixed to the actuator base or the robot joint. The wave generator is connected to the input shaft of the HDT (i.e., the electric motor side), and the flexspline is attached to the output shaft (i.e., the load or link side). The wave generator comprises a rigid elliptical disc and an outer ball bearing. The wave generator transmits torque to the flexspline by forcing it to follow the elliptical shape of the wave generator disc. Such a transmission induces a gear meshing, of a speed reduction ratio $N$, between the flexspline and the circular spline teeth.

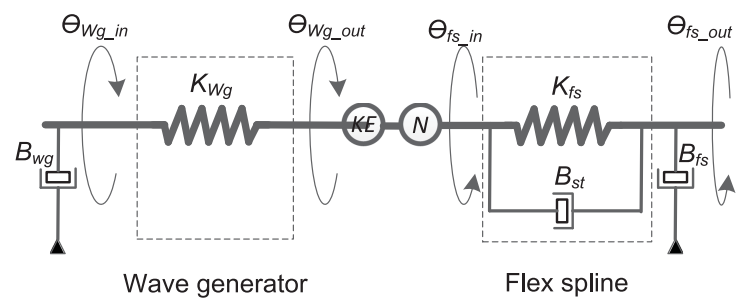

Fig. 2. Transmission model for an HDT-based robot joint, including the kinematic error (KE) torsion.

\section{A. Kinematic Model}

The HDT assembly is represented by a free body diagram in Fig. 2. At steady state conditions, the flexspline and wave generator can be considered rigid elements, where the input/output angular displacements are as defined in (1):

$$
\theta_{w g_{-} i n}=-N \theta_{f s_{-} o u t},
$$

where $\theta_{w g_{-} i n}$ is the input angular displacement to the wave generator, and $\theta_{f s-o u t}$ is the output angular displacement at the flexspline. In addition, the input/output torque is defined in (2):

$$
T_{w g \_i n}=-\frac{1}{N} T_{f s \_o u t},
$$

where $\tau_{w g_{-} i n}$ is the input torque (motor side) and $\tau_{f s_{-} o u t}$ is the output torque (link side). During transient conditions of varying speeds/loads for the HDT, both the flexspline and the wave generator are subjected to torsional deformation due to their low torsional stiffness [2], [9]. The flexspline torsional deformation $\Delta \theta_{f s}$ can be defined by the difference between the angular displacements at the flexspline terminals as in (3):

$$
\Delta \theta_{f s}=\theta_{f s_{-} \text {out }}-\theta_{f s_{-} \text {in }}
$$

where $\theta_{f s_{-} i n}$ is the angular displacement at the flexspline input terminal as shown in Fig. 2. Likewise, the wave generator torsional deformation $\Delta \theta_{w g}$ can be expressed in (4):

$$
\Delta \theta_{w g}=\theta_{w g_{\_} o u t}-\theta_{w g_{\text {in }}}
$$

where $\theta_{w g_{-} o u t}$ is the angular displacement at the wave generator output terminal. Both $\theta_{w g_{\_} \text {out }}$ and $\theta_{f s_{-} \text {in }}$ are unmeasurable internal variables at the HDT assembly. Furthermore, a third geometric torsional deformation, known as the kinematic error $\theta_{k e}$, is experienced due to the gear meshing mechanism at the flexspline [2]. The total torsional deformation between the external input and output shafts for an HDT is defined in (5):

$$
\Delta \theta=\Delta \theta_{f s}+\theta_{k e}+\frac{\Delta \theta_{w g}}{N}
$$

\section{B. Compliance Model}

There are several physical models for HDTs, depending on how the compliance and friction losses are formulated [2], [3], [9], [10]. Here, a physical model is presented and adapted from [2] for the compliance terms and for the SD term in [9].

The flexspline has a nonlinear stiffness that is a function of the flexspline torque. The flexspline torsion can be defined as in (6):

$$
\Delta \theta_{f s}=\frac{\arctan \left(C_{f s} T_{f s}\right)}{C_{f s} K_{f s 0}},
$$


where $T_{f s}$ is the elastic torque at the flexspline, and $C_{f s} \& K_{f s 0}$ are physical parameters from the manufacturer's datasheet. The $\arctan ($.$) is the arctangent function.$

The wave generator stiffness $K_{w g}$ is almost ignored by many publications, including the manufacturer's datasheet [3], [9], [11]. The main reason is related to its relatively high rigidity compared to the flexspline element. However, Zhang et al. [2] investigated an empirical form for $K_{w g}$ to model hysteresis's deformation for HDTs. The wave generator torsion is defined in (7) [2]:

$$
\Delta \theta_{w g}=\frac{\operatorname{sign}\left(T_{w g}\right)}{C_{w g} N K_{w g 0}}\left(1-e^{-C_{w g}\left|T_{w g}\right|}\right)
$$

where $T_{w g}$ is the applied torque to HDT, meaning the motor torque, and $C_{w g} \& K_{w g 0}$ are empirical parameters from a calibration test. The sign (.) is the signum function. Equation (7) provides an empirical expression for the wave generator torsional deformation $\Delta \theta_{w g}$ as a function of the motor torque $T_{w g}$.

The most recent torque estimation methods [2], [5] utilize (5)-(8) for estimating the HDT torque $T_{f s}$ as follows:

- The total torsional deformation $\Delta \theta$ is measured by the angular difference between the motor-side and link-side encoders.

- The motor torque $T_{w g}$, which is needed for calculating $\Delta \theta_{w g}$ in (7), is approximated by the motor's current measurements and the motor torque constant.

- The kinematic error $\theta_{k e}$ is calculated from a polynomial function experimentally identified as a function of the motor-side and link-side angles.

- The HDT torque $T_{f s}$ can be finally obtained by taking the inverse of (6), as in (8):

$$
T_{f s}=\frac{\boldsymbol{S}_{2} \tan \left(\Delta \theta_{f s} C_{f s} K_{f s 0} \boldsymbol{S}_{1}\right)}{C_{f s}},
$$

where $S_{1}$ and $S_{2}$ are additional empirical parameters that can be tuned to enhance the torque estimation accuracy, similar to the concept presented in [5]. However, this procedure has limitations as follows:

a) The HDT torque $T_{f s}$ is exclusively estimated by static torsional measurements $\Delta \theta_{f s}$ that don't account for the dynamic frictional losses. Most of the parameters can be obtained directly or indirectly from HDT spec sheets, and some are estimated from calibration test data of the HDT. It's true though that they need to be obtained for the torque estimation.

b) The methods in [2] and [5] are both proposed for robot joints where the motor torque can be estimated by the motor's current. They should be modified for other applications where the actuator torque cannot be easily estimated from the current.

\section{TORQue Estimation METHOD}

\section{A. Physical Principle}

The torque sensing principle is based on monitoring a frictional torque which has a good correlation to the output torque of HDT. This principle was previously utilized in [3] based on the Coulomb and viscus frictions within a full physical model for the motor's joint. Taghirad showed in [9], [12] the significance of incorporating structural damping losses for investigating high-fidelity dynamic models for HDT. However, none of the published torque estimation methods consider the structural damping contribution. Therefore, a significant measurement uncertainty has been reported due to unmapped dynamics in the compliance-based method in [5]. Taghirad defined the structural damping contribution to the HDT torque in (9):

$$
T_{f s}=K \Delta \theta_{f s}+B_{s t}\left|\Delta \dot{\theta}_{f s}\right|^{\alpha} \operatorname{sign}\left(\Delta \dot{\theta}_{f s}\right),
$$

where $\Delta \dot{\theta}_{f s}$ is the torsion deformation rate of the flexspline in $\mathrm{rad} / \mathrm{s} . B_{s t}$ is a structural damping frictional term due to the gear meshing in $\mathrm{Nm} \mathrm{s} / \mathrm{rad}$. The sign (.) is the signum function. $K, \alpha$ are empirical parameters for modeling the compliance and hysteresis contributions respectively. Here, the objective is to estimate the HDT torque by monitoring the structural damping frictional torque rather than modeling the full dynamic behavior of HDT. The total torque transmitted by the flexspline element in (9) can be decoupled into three terms: the static torsion deformation torque $\left(K \Delta \theta_{f s}\right)$, the structural damping frictional torque $\left(B_{s t} \Delta \dot{\theta}_{f s}\right)$ and an empirical term for modeling the hysteresis effects by: the power $\alpha$ of the modulus $\left(\left|\Delta \dot{\theta}_{f s}\right|^{\alpha}\right)$ and the signum function. A concept for neglecting the hysteresis effect is explained in the next subsection based on a threshold level for $\Delta \theta_{f s}$. The torque consumed by the structural damping friction is expressed in (10), as follows:

$$
T_{f s}=T_{f s 0}+\left[B_{s t} \Delta \dot{\theta}_{f s}\right] e^{-\frac{t}{\tau}},
$$

where $e^{-\frac{t}{\tau}}$ is a low pass filter of time constant $\tau . T_{f s 0}$ is the initial torque when the motor input torque is off, for example due to gravity. The filter $e^{-\frac{t}{\tau}}$ calculates the accumulated torque due to the deformation rate $\Delta \dot{\theta}_{f s}$. It is worth to mention that $\Delta \dot{\theta}_{f s}$ is independent to joint's speed and it is only influenced by the loading conditions of HDT. At the steady state loading, i.e., $\Delta \dot{\theta}_{f s}=0$ and $T_{f s} \neq 0$, the filter $e^{-\frac{t}{\tau}}$ maintains the estimated torque $T_{f s}$ with a drift error controlled by $\tau$ as shown in Fig. 3 . Depending on the application, $\tau$ can be adjusted to hold the estimated torque.

\section{B. Torque Estimation Procedure}

Step 1. Total Torsional Rate: Total HDT torsional deformation $\Delta \theta$ is calculated by subtracting the motor-side and link-side angular displacements, as in (11):

$$
\Delta \theta=\theta_{f s_{\text {out }}}-\frac{\theta_{w g_{\text {in }}}}{N},
$$

As defined in (5), the total torsion deformation $\Delta \theta$ includes the kinematic error $\theta_{k e}$ and the wave generator torsion $\Delta \theta_{w g}$. Here, we define $\theta_{\text {offset }}$ is an empirical parameter for representing the average kinematic error and the average wave generator torsion as in (12):

$$
\theta_{\text {offset }}=\theta_{k e}+\frac{\Delta \theta_{w g}}{N},
$$

The flexspline torsional rate $\Delta \dot{\theta}_{f s}$ can be calculated by applying a numerical differentiator to the total torsional deformation 

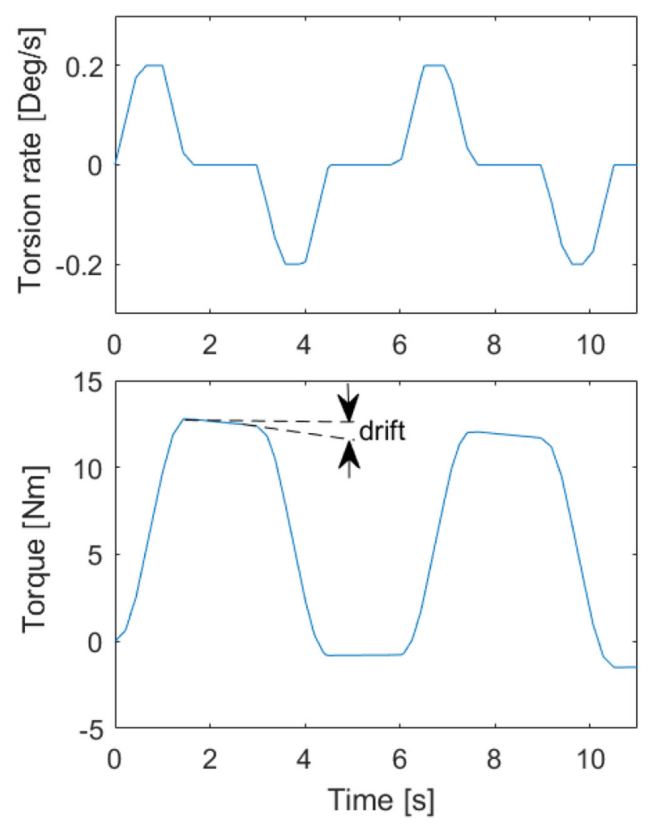

Fig. 3. Simulation example for torque estimation based on (10).

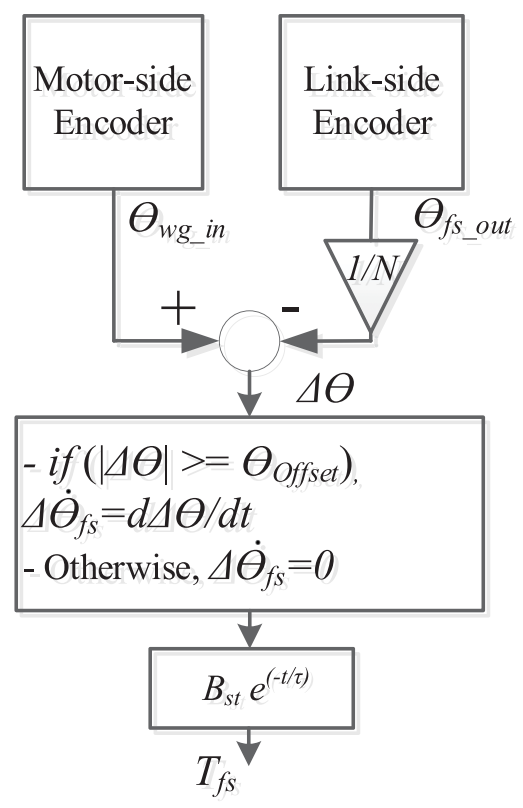

Fig. 4. Schematic diagram for the torque estimation method.

$\Delta \theta$ as defined in (13) and as shown in Fig 4.

$$
\left\{\begin{array}{ll}
\Delta \dot{\theta}_{f s}=\frac{d}{d t}(\Delta \theta), & \text { if }|\Delta \theta| \geq \theta_{\text {offset }} \\
\Delta \dot{\theta}_{f s}=0, & \text { if }|\Delta \theta|<\theta_{\text {offset }}
\end{array},\right.
$$

In (13), the torsional rate $\Delta \dot{\theta}$ is only calculated if the absolute value of $\Delta \theta$ is higher than $\theta_{\text {off set }}$. This condition is necessary for three reasons: (a) to minimize torsion contributions that are not significantly correlated to the HDT torque; (b) to avoid amplifying measurement noise which is dominated at low torsional angles; (c) to reset the drift error (Fig 3) once the torsion angle decreases below $\theta_{\text {offset }}$. The numerical differentiator could be computed with the direct discrete difference technique using the

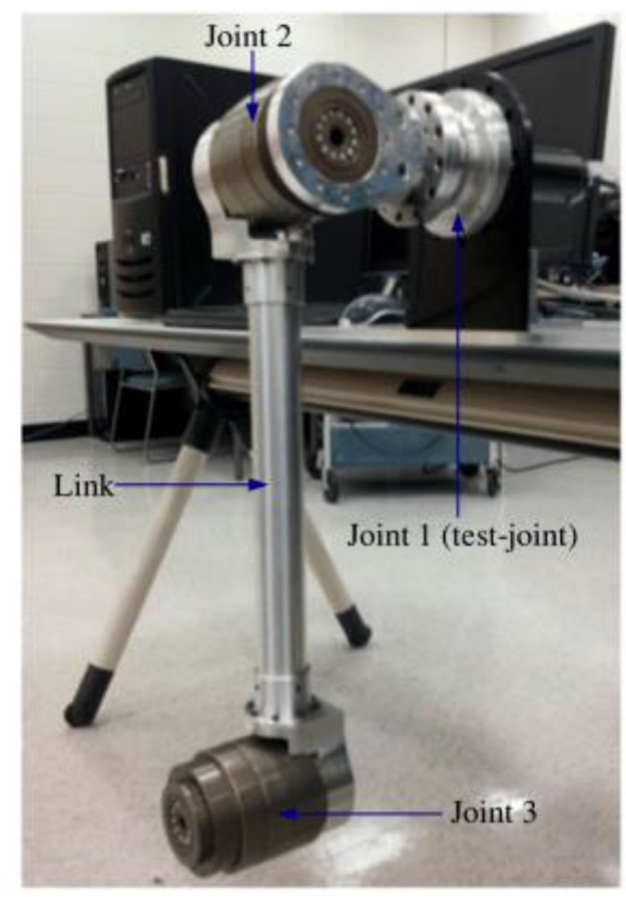

Fig. 5. A photo of the experimental setup.

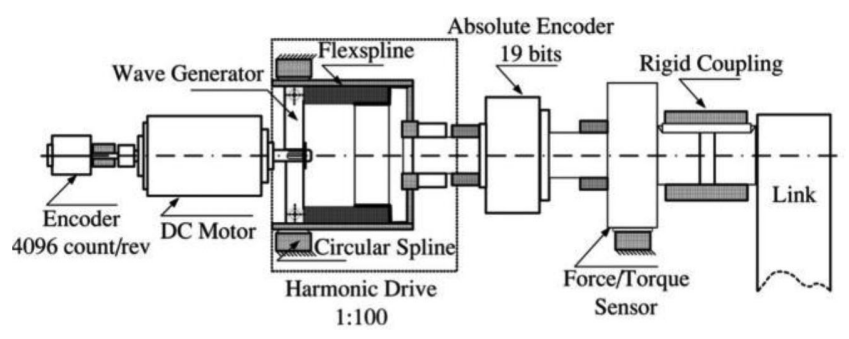

Fig. 6. Schematic diagram of the test joint [2].

MATLAB function "diff()" multiplied by the sampling rate of the measurements.

Step 2. Flexsplinetorque Model: Here, the flexspline torque model is based on the structural damping losses, which are approximated by a viscous friction coefficient $B_{s t}$ as defined in (10). The parameters $\tau, B_{s t}$ and $\theta_{\text {offset }}$ are estimated by a calibration test. The estimation objective is based on minimizing the torque error $T_{e}$ defined in (14) between the estimated torque $T_{f s}$ and a reference torque from an external torque sensor $T_{f s-s e n}$.

$$
T_{e}=\min _{\left(\tau, B_{s t}\right)}\left(T_{f s-s e n}-T_{f s}\right)
$$

\section{EXPERIMENTAL RESULTS}

\section{A. Experimental Setup}

The experimental setup has been previously used for investigating compliance-based estimation methods in [2] and [5]. The setup as shown in Fig. 5 consists of three modular joints, and Joint 1 is the test joint, which is actuated by a brushed DC motor and an HDT, model SHD-17-100-2SH, with a gear ratio of $N=100$ and a rated output torque of $16 \mathrm{Nm}$. As illustrated in Fig. 6, the test joint involves an optical incremental encoder 
TABLE I

EXPERIMENTAL SETUP PARAMETERS FOR HDT

\begin{tabular}{lcc}
\hline \multicolumn{1}{c}{ Parameter } & Unit & Value \\
\hline Rotor inertia for joint 1 & $\mathrm{kg} \mathrm{m}$ & $0.114 \times 10^{-4}$ \\
\hline Link mass & $\mathrm{kg}$ & 0.641 \\
\hline Link length & $\mathrm{m}$ & 0.45 \\
\hline Mass of joint 1 and 2 & $\mathrm{kg}$ & 1.2 \\
\hline
\end{tabular}
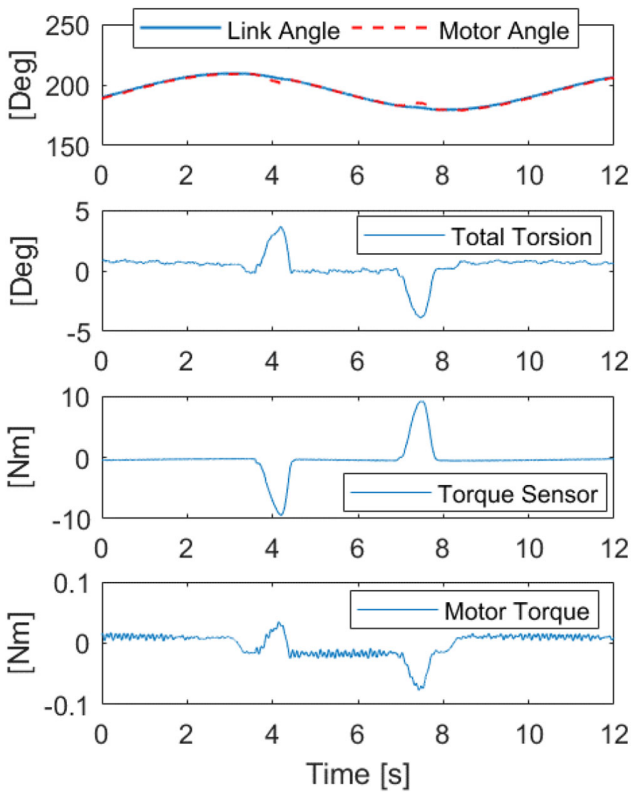

Fig. 7. The first dataset for identifying the parameters in Table II.

of 12-bit resolution attached to the motor side for measuring the wave generator angular displacement. The HDT output shaft is attached to the following robotic link via a 19-bit absolute encoder and a torque sensor, model Mini45-ERA. The physical parameters of the experimental setup are given in Table I. The parameter $T_{f s 0}=0$ because there is no initial torque prior to the motor torque.

\section{B. Results and Discussion}

The experimental verification involves two torque estimation methods: the new method that is based on the structural damping and a prior method based on a compliance model [2]. These methods are necessary for evaluating the significance of utilizing the structural damping rather than the HDT's compliance, which is the main approach used in previously published work. Two datasets obtained from the setup are used for comparison, each of which includes data for the external torque sensor, the motor torque, and the angular measurements for the motor side and link side, as shown in Fig. 7. It is worth mentioning that the motor torque is only needed for the compliance model. The first dataset is utilized for identifying the empirical parameters that are listed in Table II. The torque calibration results, including the identification errors, are shown in Fig. 8 for the structural damping and the compliance model-based methods.

There are torque ripples for the structural damping method (indicated by arrows in Fig. 8) due to the estimation discontinuity of $\Delta \dot{\theta}_{f s}$ in (13). However, these ripples have a short time
TABLE II

IDENTIFIED PARAMETERS FOR TORQUE ESTIMATION METHODS

\begin{tabular}{cccc}
\hline \multicolumn{4}{c}{ Structural damping method in (10) } \\
\hline Parameter & \multicolumn{1}{c}{ Unit } & Value \\
\hline $\boldsymbol{\tau}$ & $\mathrm{s}$ & 15.36 \\
\hline $\boldsymbol{B}_{\text {st }}$ & $\mathrm{Nm} \mathrm{s} / \mathrm{rad}$ & 3007 \\
\hline $\boldsymbol{\theta}_{\text {offset }}$ & $\mathrm{rad}$ & 0.0157 \\
\hline The compliance model based method in Eqs. (6-7) \\
\hline $\boldsymbol{K}_{\boldsymbol{f s} \mathbf{0}}$ & $\mathrm{Nm} / \mathrm{rad}$ & $8.333 \times 10^{3}$ \\
\hline $\boldsymbol{C}_{\boldsymbol{f s}}$ & $\mathrm{Nm}$ & 0.089 \\
\hline $\boldsymbol{K}_{\boldsymbol{w g} \mathbf{0}}$ & $\mathrm{Nm} / \mathrm{rad}$ & 1.33 \\
\hline $\boldsymbol{C}_{\boldsymbol{w g}}$ & $\mathrm{Nm}$ & 83.5 \\
\hline $\boldsymbol{S}_{\mathbf{1}}$ & - & 0.001 \\
\hline $\boldsymbol{S}_{\mathbf{2}}$ & - & 20 \\
\hline & &
\end{tabular}
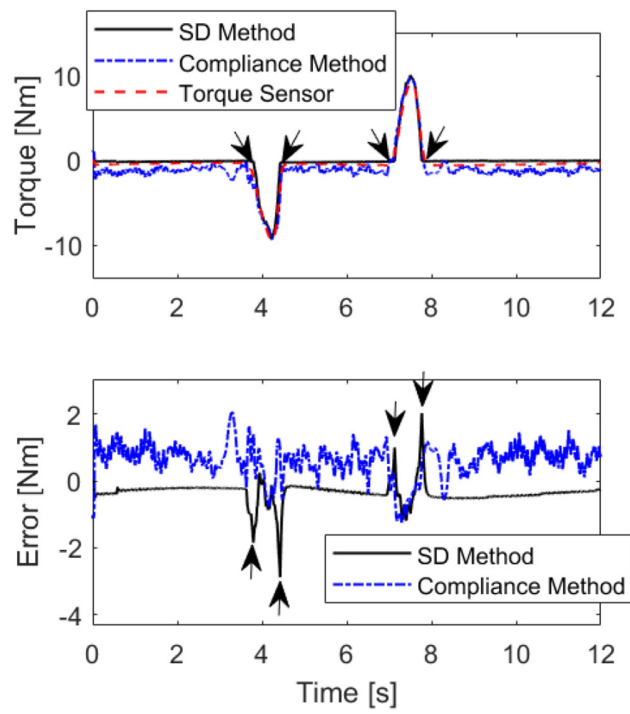

Fig. 8. Torque calibration results for the dataset in Fig. 7 including strctura damping method.

duration and they can be minimized by a moving average filter. The second dataset, shown in Fig. 9, is used for evaluating the efficiency of the torque estimation methods.

As shown in Fig. 10, the average torque errors for the compliance and structural damping methods are $0.6 \mathrm{Nm}$ and $-0.05 \mathrm{Nm}$, respectively. While the maximum torque errors are $3.7 \mathrm{Nm}$ and $2.3 \mathrm{Nm}$ for the compliance and the structural damping methods, the estimated structural damping torque is smoother than the compliance method because it involves the simplified first-order model in (10). Please note that the results of the compliance method in Fig 10 do not include data smoothing or filtration, compared to the results in [2], in order to avoid adding additional adjustable parameters. The torque results for the compliance method are subjected to noises from the motor torque, the nonlinear formula for the wave generator torsion in (7), and the dynamic frictional losses.

The torque estimation for both methods is approximately similar as that for a torque range higher than $2 \mathrm{Nm}$. Nevertheless, the structural damping method provides several advantages compared to the compliance method. The structural damping method uses only three simple empirical parameters rather than 

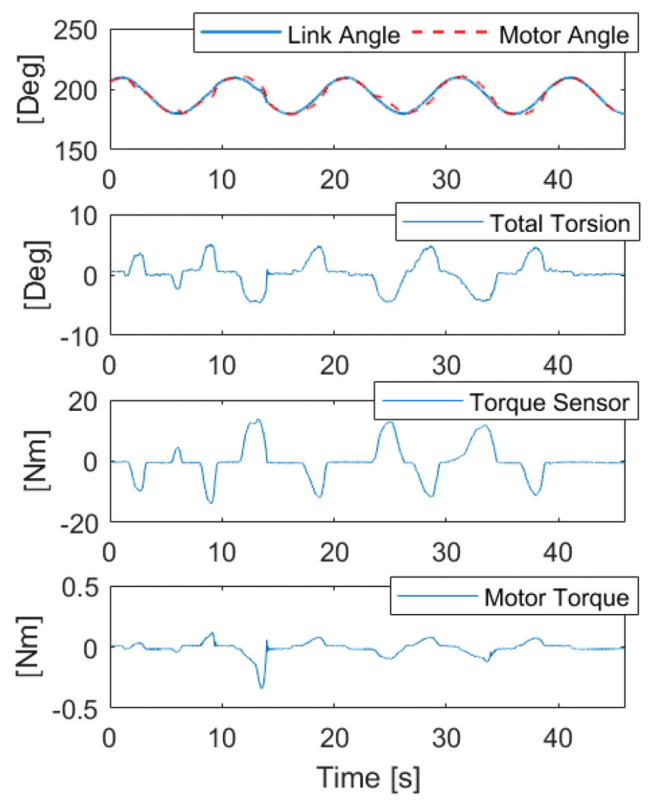

Fig. 9. The second dataset for evaluating torque estimation performance.
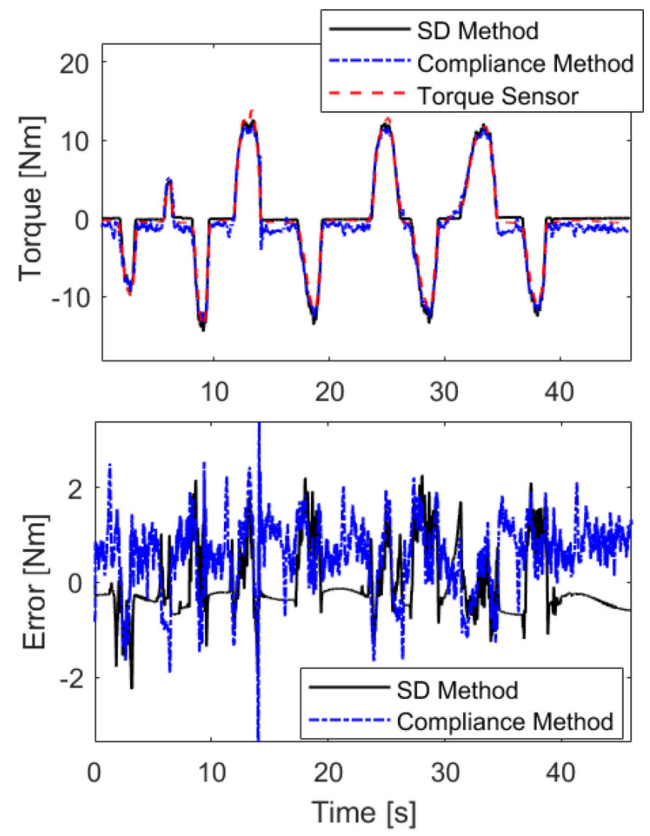

Fig. 10. Torque estimation results for the dataset in Fig. 8.

the six empirical and physical parameters for the compliance model-based method.

On one side, the structural damping method is based on a first-order model and the hysteresis is minimized by using the torsional rate. The structural damping method is mainly developed for dynamic applications of short static loading conditions. On the other side, the compliance method is based on a nonlinear static model using direct torsion measurements. Such a static model does not account for dynamical losses, and the motor torque measurements are necessary for compensating the hysteresis error. The compliance-based method outperforms the structural damping method for nondynamic applications where the HDT torque is almost in static loading conditions.

\section{CONCLUSION}

A torque estimation method has been developed and tested on a robotic joint with an HDT. The method is based on monitoring the structural damping friction for the flexspline, an elastic element inside the HDT assembly. The HDT's torsion is calculated in a manner similar to the existing methods, by the angular encoders at the input/output shafts of the HDT. A numerical differentiator is used to convert the torsion data into the torsional rate. A simplified linear dynamic model is empirically identified for estimating the HDT torque based on the torsional rate measurements. The structural damping method provides several advantages in comparison to compliance-based torque estimation methods, which are related to minor hysteresis errors, a lower computational effort, and a minimum number of sensor measurements. The structural damping method does not need the motor torque measurements for compensating the hysteresis errors. Alternatively, the performance of the structural damping method for dynamic applications is based on using the torsion rate for sensing the HDT torque, while the static hysteresis is fundamentally avoided. The compliance-based method outperforms the structural damping method for nondynamic applications where the HDT torque is almost in static loading conditions. The experimental results showed enhanced torque estimation performance of the structural damping method over the compliance method for dynamic applications using the same setup.

\section{REFERENCES}

[1] K. Ueura and R. Slatter, "Development of the harmonic drive gear for space applications," in Proc. 8th Eur. Symp., 1999, pp. 259-264.

[2] H. Zhang, S. Ahmad, and G. Liu, "Torque estimation for robotic joint with harmonic drive transmission based on position measurements," IEEE Trans. Robot., vol. 31, no. 2, pp. 322-330, Apr. 2015.

[3] D. Vassileva, Y. Kiyosawa, and M. Suzuki, "Sensorless torque control for a robot with harmonic drive reducers," Mech. Based Des. Struct. Mach., vol. 39, no. 2, pp. 253-267, 2011.

[4] S. Katsura, Y. Matsumoto, and K. Ohnishi, "Analysis and experimental validation of force bandwidth for force control," IEEE Trans. Ind. Electron., vol. 53, no. 3, pp. 922-928, Jun. 2006.

[5] K. Xia, L. Ding, G. Liu, H. Gao, and Z. Deng, "A novel virtual torque sensor for rescue robots with harmonic drives," Preprints, 2016, Art. no. 2016090084.

[6] M. Ismail, "Fault tolerant electro-mechanical actuation architectures for unmanned aerial vehicles," Internal Report, German Aerospace Center, Institute of Flight Systems, Apr. 2020.

[7] H. D. Taghirad and P. R. Belanger, "Intelligent built-in torque sensor for harmonic drive systems," IEEE Trans. Instrum. Meas., vol. 48, no. 6, pp. 1201-1207, 1999.

[8] J. W. Sensinger and R. F. Weir, "Improved torque fidelity in harmonic drive sensors through the union of two existing strategies," IEEE/ASME Trans. Mechatron., vol. 11, no. 4, pp. 457-461, Aug. 2006.

[9] H. D. Taghirad, "On the modelling and identification of harmonic drive systems," Centre for Intelligent Machines, McGill University, 1997.

[10] C. Preissner, T. J. Royston, and D. Shu, "A high-fidelity harmonic drive model," J. Dyn. Syst. Meas. Control, vol. 134, no. 1, 2012, Art. no. 011002.

[11] Harmonic Drive GmbH. Lahn, Germany: Engineering Data Catalog CSD2A, Nov. 2014.

[12] H. D. Taghirady and P. R. Belanger, "A nonlinear model for harmonic drive friction and compliance," in Proc. IEEE Int. Conf. Robot. Automat., 1998, pp. 1-6. 